PROCEEDINGS OF THE

AMERICAN MATHEMATICAL SOCIETY

Volume 132, Number 11, Pages 3225-3229

S 0002-9939(04)07567-7

Article electronically published on June 21, 2004

\title{
EXACT LOCAL BEHAVIOR OF POSITIVE SOLUTIONS FOR A SEMILINEAR ELLIPTIC EQUATION WITH HARDY TERM
}

\author{
JIANQING CHEN
}

(Communicated by David S. Tartakoff)

\begin{abstract}
We characterize an exact growth order near zero for positive solutions of a semilinear elliptic equation with Hardy term. This result strengthens an existence result due to E. Jannelli [The role played by space dimension in elliptic critical problems, JDE 156 (1999), 407-426].
\end{abstract}

\section{INTRODUCTION}

This paper is concerned with the exact local behavior of solutions for the following elliptic equation:

$$
\left\{\begin{aligned}
-\Delta u-\frac{\mu}{|x|^{2}} u & =f(x, u) & & \text { in } \quad \Omega \backslash\{0\}, \\
u(x) & >0 & & \text { in } \Omega \backslash\{0\}, \\
u(x) & =0 & & \text { on } \partial \Omega,
\end{aligned}\right.
$$

where $0 \in \Omega \subset \mathbb{R}^{N}(N \geq 3)$ is a bounded domain with smooth boundary, $0 \leq \mu<$ $\bar{\mu}=\left(\frac{N-2}{2}\right)^{2}$ and $\bar{\mu}$ is the best constant in the Hardy inequality.

The starting point is the excellent paper due to Jannelli $[8$, where the author proved, among other results, that when $f(x, u)=u^{2^{*}-1}+\lambda u\left(2^{*}=\frac{2 N}{N-2}\right)$,

- if $0 \leq \mu<\bar{\mu}-1$, then $(P)$ has at least one solution $u \in H_{0}^{1}(\Omega)$ provided $0<\lambda<\lambda_{1}(\mu)$

- if $\mu \geq 0$ and $\bar{\mu}-1<\mu<\bar{\mu}$, then $(P)$ has at least one solution $u \in H_{0}^{1}(\Omega)$ provided $\lambda_{*}(\mu)<\lambda<\lambda_{1}(\mu)$ for some $\lambda_{*}(\mu)>0$,

where $\lambda_{1}(\mu)$ is the first eigenvalue of the operator $-\Delta-\frac{\mu}{|x|^{2}}$ with Dirichlet boundary condition. For other existence results concerning the variant problem of $(P)$, we refer the interested reader to [3, 6, 9, 10, 11] and the references therein.

From the results and the references mentioned above, we know nothing about further properties of solutions of $(P)$. The main purpose of the present paper is to give an exact local behavior of solutions of $(P)$. Before stating the main result, we formulate our assumptions on $f$ and clarify some terminology. Throughout this

Received by the editors May 13, 2003.

2000 Mathematics Subject Classification. Primary 35J65, 58E05.

Key words and phrases. Positive solutions, exact local behavior, critical Hardy-Sobolev exponent.

The author was supported by the National Natural Sciences Foundation of China.

(C)2004 American Mathematical Society 
paper, we assume that

$(F): f(x, t): \Omega \times \mathbb{R}^{+} \rightarrow \mathbb{R}^{+}$is continuous and locally Lipschitz continuous with respect to $t$. Moreover,

$$
f(x, t) \leq C\left(|t|^{2^{*}-1}+|t|\right), \quad \text { for some } C>0 .
$$

We say that $u \in H_{0}^{1}(\Omega)$ is a weak solution of $(P)$ if for any $\phi \in H_{0}^{1}(\Omega)$, it follows that

$$
\int_{\Omega}\left(\nabla u \nabla \phi-\frac{\mu}{|x|^{2}} u \phi\right) d x=\int_{\Omega} f(x, u) \phi d x .
$$

Indeed, the assumptions on $f$ and the standard elliptic regularity theory imply that $u \in C^{2}(\Omega \backslash\{0\})$. In other words, if $u \in H_{0}^{1}(\Omega)$ satisfies (1.1) for any $\phi \in H_{0}^{1}(\Omega)$, then $u$ also satisfies $(P)$ in the classical sense.

Our main result reads as follows:

Theorem 1.1. Suppose that hypothesis $(F)$ holds. If $u \in H_{0}^{1}(\Omega)$ is a solution of $(P)$, then there exist positive constants $M_{1}$ and $M_{2}$ such that

$$
M_{1}|x|^{-(\sqrt{\mu}-\sqrt{\bar{\mu}-\mu})} \leq u(x) \leq M_{2}|x|^{-(\sqrt{\mu}-\sqrt{\bar{\mu}-\mu})} \quad \text { for any } x \in B_{r}(0) \backslash\{0\}
$$

holds for $r$ sufficiently small.

Remark 1.2. (i) When $\mu=0$, Theorem 1.1 implies that $u(0)$ is positive, and we come back to the usual case. We also want to mention that some other kind of asymptotic behavior has been obtained in [1] under some additional assumptions on $f$ to the case of $\mu=0$.

(ii) When $0<\mu<\bar{\mu}, f(x, u)=u^{2^{*}-1}, \Omega=\mathbb{R}^{N}$, it is shown in 11] that all positive solutions of $(P)$ can be written as

$$
U_{\varepsilon}(x)=\frac{[4 \varepsilon(\bar{\mu}-\mu) N /(N-2)]^{\frac{N-2}{4}}}{\left[\varepsilon|x|^{\gamma^{\prime} / \sqrt{\mu}}+|x|^{\gamma / \sqrt{\mu}}\right]^{\frac{N-2}{2}}}, \quad \forall \varepsilon>0,
$$

where $\gamma^{\prime}=\sqrt{\bar{\mu}}-\sqrt{\bar{\mu}-\mu}, \gamma=\sqrt{\bar{\mu}}+\sqrt{\bar{\mu}-\mu}$. The estimate of singularity of positive solutions of $(P)$ at zero coincides with the singularity of $U_{\varepsilon}(x)$ at zero.

(iii) From Theorem 1.1, we know that any positive solutions of $(P)$ have stronger and stronger singularity as $\mu \rightarrow \bar{\mu}$. Moreover, the singularity is completely determined by the operator $-\Delta-\frac{\mu}{|x|^{2}}$.

Remark 1.3. We believe that the characterization of local behavior in Theorem 1.1 can help to find multiple solutions of a variant of problem $(P)$. This is another work in preparation [4].

Notations: Throughout this paper, $H_{0}^{1}(\Omega), L^{p}(\Omega)$ are standard Sobolev spaces with standard norm $\|\cdot\|,|\cdot|_{p} . L^{p}\left(\Omega,|x|^{t} d x\right)$ denotes the weighted Sobolev space with norm $|\cdot|_{p, t}$. All integrals are taken over $\Omega$ unless stated otherwise. $C$ will denote various positive constants whose exact values are not important.

\section{Proof of the MAIN ReSUlt}

In this section, we will prove Theorem 1.1. The method is the Moser iteration, which has been used before; see, e.g., [5, 7]. Here we will borrow an idea from [5]. The novelty is that we can characterize the exact local behavior of positive solutions independently of the explicit form of $f$ and that the proof is direct. Before giving the proof of Theorem 1.1, we make some preparations. 
Suppose that $u \in H_{0}^{1}(\Omega)$ is a solution of $(P)$. Then as we pointed out before, $u$ also satisfies $(P)$ in the classical sense, and $u \in C^{2}(\Omega \backslash\{0\})$. Let $u(x)=|x|^{s} w(x)$, where $s=-(\sqrt{\bar{\mu}}-\sqrt{\bar{\mu}-\mu})$. Direct computation shows that $w(x)$ satisfies

$$
-|x|^{s} \Delta w-2 s|x|^{s-2}\langle x, \nabla w\rangle=f\left(x,|x|^{s} w(x)\right), \quad x \in \Omega \backslash\{0\}
$$

in the classical sense and $w \in C^{2}(\Omega \backslash\{0\})$. Multiplying (2.1) by $|x|^{s}$, we have that

$$
-\operatorname{div}\left(|x|^{2 s} \nabla w(x)\right)=|x|^{s} f\left(x,|x|^{s} w(x)\right), \quad x \in \Omega \backslash\{0\}
$$

holds in the classical sense. Now we have the following proposition.

Proposition 2.1. If $w \in C^{2}(\Omega \backslash\{0\})$ is positive and satisfies (2.2), then $w \in$ $L^{\infty}\left(B_{r}(0)\right)$ for $r>0$ small.

Proof. Let $\eta$ be a cut-off function in $B_{R}(0), R>r$. Multiplying (2.2) by $\psi=$ $\eta^{2} w \min \left\{w^{2 \gamma}, L^{2}\right\}(\gamma>0)$ and integrating by parts, we get

$$
\int|x|^{2 s} \nabla w \nabla \psi d x=\int|x|^{s} f\left(x,|x|^{s} w(x)\right) \psi d x .
$$

Since

$$
\nabla \psi=2 \eta w \min \left\{w^{2 \gamma}, L^{2}\right\} \nabla \eta+\eta^{2} \min \left\{w^{2 \gamma}, L^{2}\right\} \nabla w+2 \gamma \eta^{2} w^{2 \gamma}
$$

holds on the set $\left\{x ; w^{\gamma} \leq L\right\}$, by letting $\xi(x)=\eta w \min \left\{w^{\gamma}, L\right\}$, we have

$$
\begin{aligned}
\int|x|^{2 s}|\nabla \xi|^{2} d x & \leq C \gamma\left(\int|x|^{2 s}|\nabla \eta|^{2} w^{2} \min \left\{w^{2 \gamma}, L^{2}\right\} d x\right. \\
& \left.+\int|x|^{s} f\left(x,|x|^{s} w(x)\right) \eta^{2} w \min \left\{w^{2 \gamma}, L^{2}\right\} d x\right) .
\end{aligned}
$$

Using assumption $(F)$, we can get

$$
\begin{aligned}
\int|x|^{2 s}|\nabla \xi|^{2} d x & \leq C \gamma\left(\int|x|^{2 s}|\nabla \eta|^{2} w^{2} \min \left\{w^{2 \gamma}, L^{2}\right\} d x\right. \\
& +\int|x|^{2^{*} s} \eta^{2} w^{2^{*}} \min \left\{w^{2 \gamma}, L^{2}\right\} d x \\
& \left.+\int|x|^{2 s} \eta^{2} w^{2} \min \left\{w^{2 \gamma}, L^{2}\right\} d x\right) .
\end{aligned}
$$

By the Hölder inequality, we obtain that

$$
\begin{gathered}
\int|x|^{2^{*} s} \eta^{2} w^{2^{*}} \min \left\{w^{2 \gamma}, L^{2}\right\} d x \leq\left(\int|x|^{2^{*} s} \xi^{2^{*}} d x\right)^{2 / 2^{*}}\left(\int_{B_{R}(0)}|x|^{2^{*} s} w^{2^{*}} d x\right)^{1-2 / 2^{*}} \\
\int|x|^{2 s} \eta^{2} w^{2} \min \left\{w^{2 \gamma}, L^{2}\right\} d x \leq\left(\int|x|^{2^{*} s} \xi^{2^{*}} d x\right)^{2 / 2^{*}}\left|B_{R}(0)\right|^{1-2 / 2^{*}} .
\end{gathered}
$$

From the weighted Sobolev inequality (see, e.g., [2]), we have that

$$
\left(\int|x|^{2^{*} s} \xi^{2^{*}} d x\right)^{2 / 2^{*}} \leq C \int|x|^{2 s}|\nabla \xi|^{2} d x .
$$

Since $\operatorname{supp} \eta(x) \subset B_{R}(0)$, we can choose $R$ small enough such that

$$
\left(\int_{B_{R}(0)}|x|^{2^{*} s} w^{2^{*}} d x\right)^{1-2 / 2^{*}}<\frac{1}{4 C \gamma} \quad \text { and } \quad\left|B_{R}(0)\right|^{1-2 / 2^{*}}<\frac{1}{4 C \gamma} .
$$


Therefore,

$$
\begin{aligned}
\left(\int|x|^{2^{*} s} \xi^{2^{*}} d x\right)^{2 / 2^{*}} & \leq C \gamma \int|x|^{2 s}|\nabla \eta|^{2} w^{2} \min \left\{w^{2 \gamma}, L^{2}\right\} d x \\
& \leq C \gamma \int|x|^{2 s}|\nabla \eta|^{2} w^{2(\gamma+1)} d x .
\end{aligned}
$$

Taking $\gamma+1=2^{*} / 2$ and $\eta$ to be constant near zero and letting $L$ go to infinity, we get that $w \in L^{2^{*}}\left(\Omega,|x|^{2^{*} s} d x\right)$.

Now let $\eta$ be a cut-off function in $B_{r+r_{0}}$ for $r$ sufficiently small such that $|\nabla \eta|<$ $\frac{C}{r_{0}}, \eta \equiv 1$ on $B_{r}(0)$. Taking $0<t<2^{*}-2$ and by the Hölder inequality we have that

$$
\begin{aligned}
\int_{B_{r+r_{0}}}|x|^{2 s}|\nabla \eta|^{2} w^{2(\gamma+1)} d x \leq & \frac{C}{r_{0}^{2}}\left(\int_{B_{r+r_{0}}}\left(|x|^{(2+t) s} w^{2(\gamma+1)}\right)^{\frac{2^{*}}{2+t}} d x\right)^{\frac{2+t}{2^{*}}} \\
& \times\left(\int_{B_{r+r_{0}}}|x|^{-t s \cdot \frac{2^{*}}{2^{*}-2-t}} d x\right)^{\frac{2^{*}-2-t}{2^{*}}} .
\end{aligned}
$$

It follows from $\int_{B_{r+r_{0}}}|x|^{-t s \cdot \frac{2^{*}}{2^{*}-2-t}} d x<\infty$ that

$$
\int_{B_{r+r_{0}}}|x|^{2 s}|\nabla \eta|^{2} w^{2(\gamma+1)} d x \leq \frac{C}{r_{0}^{2}}\left(\int_{B_{r+r_{0}}}\left(|x|^{(2+t) s} w^{2(\gamma+1)}\right)^{\frac{2^{*}}{2+t}} d x\right)^{\frac{2+t}{2^{*}}} .
$$

Denoting $\gamma+1=\chi^{j}, \chi=\frac{2+t}{2}, r_{0}=2^{-j}, j=1,2, \ldots$, we have

$$
\left(\int_{B_{r+r_{0}}}|x|^{2^{*} s} w^{\chi^{j} \cdot 2^{*}} d x\right)^{\frac{2}{2^{*}}} \leq \frac{C(\gamma+1)}{r_{0}^{2}}\left(\int_{B_{r+r_{0}}}|x|^{2^{*} s} w^{\chi^{j-1} \cdot 2^{*}} d x\right)^{\frac{2+t}{2^{*}}} .
$$

Therefore, replacing $\gamma$ by $\chi^{j}-1$ and using (2.5) recursively, we get

$$
\left(\int_{B_{r}}|x|^{2^{*} s} w^{\chi^{j} \cdot 2^{*}} d x\right)^{\frac{1}{\chi^{j} \cdot 2^{*}}} \leq C^{\sum_{k=1}^{j} \frac{1}{2 \chi^{k}}} \chi^{\sum_{k=1}^{j} \frac{k}{2 \chi^{k}}} 2^{\sum_{k=1}^{j} \frac{k}{\chi^{k}}}\left(\int_{B_{r+\frac{1}{2}}}|x|^{2^{*} s} w^{2^{*}} d x\right)^{\frac{2+t}{2 \cdot 2^{*}}} .
$$

Since the infinite sum in the right-hand side converges, we obtain that $w(x)$ is bounded in $B_{r}(0)$ by letting $j$ goes to infinity. The proof is complete.

Proposition 2.2. If $w \in C^{2}(\Omega \backslash\{0\})$ is positive and satisfies (2.2), then there exists $r_{1}>0, r_{1}$ may be small, such that

$$
w(x) \geq \min _{|x|=r_{1}} w(x)=C_{0}>0 \quad \text { for any } x \in B_{r_{1}}(0) .
$$

Proof. Let $\phi(t)=\min _{|x|=t} w(x)$. For any $0<t_{1}<t_{2}<r(r$ is chosen as in Proposition [2.1), we define a comparison function $g(x)=A|x|^{2-N-2 s}+B$, where $A$ and $B$ are such that $g(x)=\phi\left(t_{i}\right)$ for $|x|=t_{i}, i=1,2$. More precisely, we have

$$
A=\frac{\phi\left(t_{2}\right)-\phi\left(t_{1}\right)}{t_{2}^{2-N-2 s}-t_{1}^{2-N-2 s}}, \quad B=\frac{\phi\left(t_{2}\right) t_{1}^{2-N-2 s}-\phi\left(t_{1}\right) t_{2}^{2-N-2 s}}{t_{1}^{2-N-2 s}-t_{2}^{2-N-2 s}} .
$$

Since $\operatorname{div}\left(|x|^{2 s} \nabla w\right) \leq 0$ for $x \in \Omega \backslash\{0\}$, we have $\operatorname{div}\left(|x|^{2 s} \nabla(w(x)-g(x)) \leq 0\right.$, while from the definition of $w(x)$, we know that $w(x)-g(x) \geq 0$ in $\partial\left(B_{t_{2}}(0) \backslash B_{t_{1}}(0)\right)$. 
The maximum principle implies that $w(x) \geq g(x)$ in $B_{t_{2}}(0) \backslash B_{t_{1}}(0)$. In other words, denoting $a=t^{2-N-2 s}$, we have that

$$
\begin{aligned}
w(x)_{|x|=t} & \geq g(x)_{|| x \mid=t}=\frac{\phi\left(t_{2}\right)-\phi\left(t_{1}\right)}{a_{2}-a_{1}} a+\frac{\phi\left(t_{1}\right) a_{2}-\phi\left(t_{2}\right) a_{1}}{a_{2}-a_{1}} \\
& =\frac{a_{2}-a}{a_{2}-a_{1}} \phi\left(t_{1}\right)+\frac{a-a_{1}}{a_{2}-a_{1}} \phi\left(t_{2}\right) .
\end{aligned}
$$

It follows that $w(x) \geq \min _{|x|=r_{1}} w(x)=C_{0}>0$ for some $r_{1}<r$ and any $x \in B_{r_{1}}(0)$. We end the proof.

Proof of Theorem [1.1. Keep the relation $u(x)=|x|^{s} w(x)$ in mind. On the one hand, we get from Proposition 2.1 that

$$
u(x)=|x|^{s} w(x) \leq M_{2}|x|^{-(\sqrt{\mu}-\sqrt{\mu-\mu})}, \quad \forall x \in B_{r}(0) \backslash\{0\} .
$$

On the other hand, Proposition 2.2 implies that

$$
u(x)=|x|^{s} w(x) \geq|x|^{s} \min _{|x|=r_{1}} w(x)=M_{1}|x|^{-(\sqrt{\mu}-\sqrt{\bar{\mu}-\mu})}
$$

for any $x \in B_{r_{1}}(0) \backslash\{0\}$. (Here $r$ can be chosen sufficiently small if necessary.) Thus we complete the proof.

\section{ACKNOWLEDGMENT}

The author would like to express his thanks to the unknown referee for many valuable comments and suggestions, to Prof. S. Li and Prof. Y. Li for constant help and encouragement, and to Dr. S. Chen for useful conversations.

\section{REFERENCES}

[1] L. Caffarelli, B. Gidas and J. Spruck, Asymptotic symmetry and local behaviour of semilinear elliptic equations with critical Sobolev growth, Comm. Pure Appl. Math., 42 (1989), 271-297. MR 90c:35075

[2] L. Caffarelli, R. Kohn and L. Nirenberg, First order interpolation inequalities with weights, Compositio Math., 53 (1984), 259-275. MR 86c:46028

[3] J. Chen, Existence of solutions for a nonlinear PDE with an inverse square potential, J. Differential Equations 195 (2003), 497-519.

[4] J. Chen, Multiple positive solutions for a class of nonlinear elliptic equation, J. Math. Anal. Appl., to appear.

[5] K. S. Chou and C. W. Chu, On the best constant for a weighted Sobolev-Hardy inequality, J. London Math. Soc., 48 (1993), 137-151. MR 94h:46052

[6] A. Ferrero and F. Gazzola, Existence of solutions for singular critical growth semilinear elliptic equations, J. Differential Equations, 177 (2001), 494-522. MR 2002m:35068

[7] Q. Han and F. Lin, Elliptic Partial Differential Equations, Courant Lecture Notes in Mathematics 1, New York University, 1997. MR 2001d:35035

[8] E. Jannelli, The role played by space dimension in elliptic critical problems, J. Differential Equations 156 (1999), 407-426. MR 2000f:35053

[9] D. Smets, Nonlinear Schrödinger equations with Hardy potential and critical nonlinearities, Preprint of Université Catholique de Louvain, Institut de Mathématique Pure et Appliquée, Chemin du Cyclotron 2, Belgium, (2001).

[10] D. Smets and A. Tesei, On a class of singular elliptic problems with first order terms, Adv. Diff. Equations, Vol. 8 (2003), No. 3, 257-278.

[11] S. Terracini, On positive entire solutions to a class of equations with singular coefficient and critical exponent, Adv. Diff. Equations Vol. 1 (1996), 241-264. MR 97b:35057

Department of Mathematics, Fujian Normal University, Fuzhou, 350007, Peoples RePUBliC OF CHINA

E-mail address: jqchen@fjnu.edu.cn 